\title{
Closed-wedge Patelloplasty for the Treatment of Distal Patellofemoral Maltracking and Instability due to Severe Patellar Dysplasia: Case Report and Surgical Technique
}

\author{
Jannik Frings ${ }^{1}$, Fabian Freudenthaler ${ }^{2}$, Matthias Krause ${ }^{3}$, Karl-Heinz Frosch ${ }^{4}$
}

\begin{abstract}
Background: Patellofemoral maltracking is caused by different anatomical factors. Most of them are associated with a proximal maltracking, which alters the patella's engagement into the trochlear groove and predisposes the patellofemoral joint for instability. Different surgical techniques have been described to realign patellar tracking, however, most of which address proximal patellar maltracking.

Aim: The aim of this article is to demonstrate the influence of patella-related deformities on patellar tracking and to present a novel surgical technique for the treatment of distal patellar maltracking, caused by a severe patellar dyplasia.

Case Description: We report the case of a 23-year-old patient with a severe patellar dysplasia, presenting a distal patellar maltracking with recurring dislocations in deep flexion. Due to her instability, the patient was immobilised and dependent on the constant use of walking aids. Radiological images showed a concavely shaped patellar, which articulated exclusively with the lateral epicondyle and caused the patella to dislocate laterally, starting at a flexion angle of $60^{\circ}$. An anterior closing-wedge osteotomy was used to reshape and recenter the patella, which was complemented by a medial patellofemoral ligament reconstruction. At the 18-month follow-up, the patient presented pain free and fully remobilised, without the use of walking aids. Patellar tracking was reestablished, with a possible knee flexion until $140^{\circ}$. No redislocation of the patella had occurred.

Conclusion: Distal patellofemoral maltracking, caused by a severe patellar dysplasia, can successfully be treated with an anterior closed-wedge osteotomy of the patella. In combination with a medial patellofemoral ligament reconstruction, patellofemoral stability can be reestablished, to prevent further dislocations.

Clinical Significance:There are multiple factors, which may cause patellar maltracking. A thorough clinical and radiological preoperative analysis is mandatory, in order to clearly identify the underlying pathologies, as these may affect patellar tracking proximally or distally.

Keywords: Distal maltracking, Instability, Maltracking, Osteotomy, Patella, Patelloplasty.

Strategies in Trauma and Limb Reconstruction (2020): 10.5005/jp-journals-10080-1501
\end{abstract}

\section{BACKGROUND}

Patellofemoral instability has been shown to be associated with a variety of predisposing factors, such as the patient's age, a history of contralateral dislocations, skeletal immaturity or variations of normal anatomy. ${ }^{1-7}$ Besides instability, which is defined by recurring dislocations, physiological patella tracking - in relation to the trochlear groove - is, in many cases, pathologically altered by the presence of predisposing anatomic risk factors. ${ }^{5}$ These typically include lateral soft-tissue contractions or muscular imbalance, patella alta, ${ }^{8}$ a pathologically lateralised tibial tubercle, ${ }^{9-11}$ trochlear dysplasia, ${ }^{12,13}$ genu valgum, ${ }^{2,4}$ or torsional deformities of the femur or tibia. ${ }^{3,5,14}$

Yet, all of the above mainly influence patellar engagement proximal to the trochlear groove. However, if patellar tracking is impaired distally to the trochlear groove, the influence of a hypo- or dysplastic patella needs to be considered when planning surgical realignment. ${ }^{15-17}$ Accordingly, the surgical correction of a proximal maltracking cannot be expected to deliver satisfying results if a distally pronounced maltracking is observed.

We report a case of distal patellofemoral maltracking caused by a severely dysplastic patella infera. Therefore, we present a novel technique of patellofemoral realignment by anterior closed-wedge patelloplasty, combined with a proximalisation of the tibial tubercle and the reconstruction of the medial patellofemoral ligament (MPFL). To the best of our knowledge, such a technique has not yet been described in the current literature.
${ }^{1,3}$ Department of Trauma and Orthopaedic Surgery, University Medical Center Hamburg-Eppendorf, Hamburg, Germany

${ }^{2}$ Department of Orthopaedic Surgery, Schoen Klinik Eilbek, Hamburg, Germany

${ }^{4}$ Department of Trauma and Orthopaedic Surgery, SportsTraumatology, BG Hospital Hamburg, Hamburg, Germany; Department of Trauma and Orthopaedic Surgery, University Medical Center Hamburg-Eppendorf, Hamburg, Germany

Corresponding Author: Jannik Frings, Department of Trauma and Orthopaedic Surgery, University Medical Center Hamburg-Eppendorf, Hamburg, Germany, Phone: +49 40 741053459, e-mail: j.frings@uke.de How to cite this article: Frings J, Freudenthaler F, Krause $M$, et al. Closed-wedge Patelloplasty for the Treatment of Distal Patellofemoral Maltracking and Instability due to Severe Patellar Dysplasia: Case Report and Surgical Technique. Strategies Trauma Limb Reconstr 2020;15(3):184-192.

Source of support: Nil

Conflict of interest: None

\section{Case Description}

A 23-year-old female patient with massive chronic anterior knee pain was referred to our department, five years after a medialising 

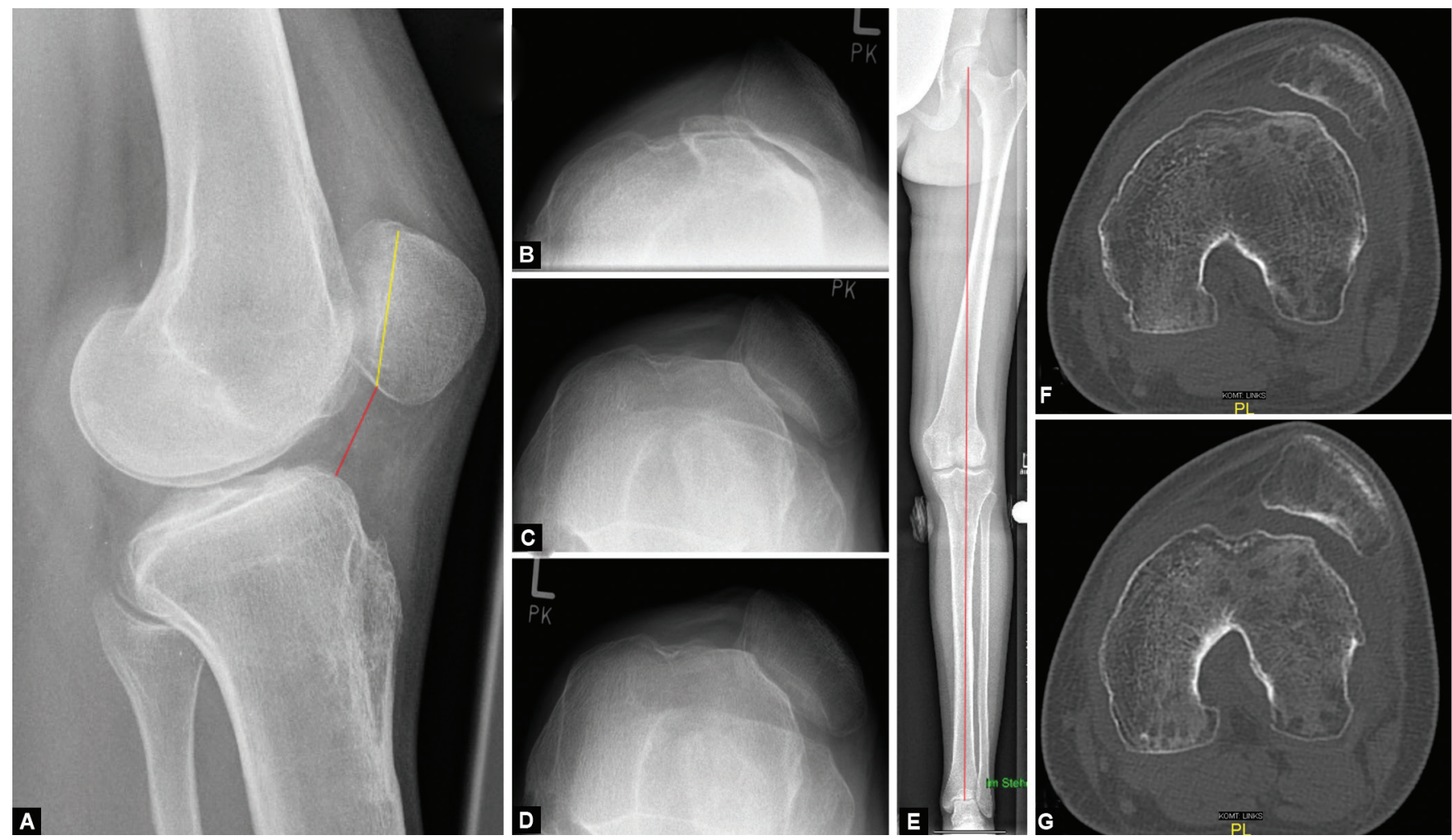

Figs. $1 \mathrm{~A}$ to G: A lateral radiograph of the left knee showed a patella infera, with a CDI of $0.74(\mathrm{~A})$, while the leg axis in the frontal plane was found to be straight in the standing long-leg radiograph (E). On tangential radiographs of the patella in 30,60 and $90^{\circ}$ of knee flexion, the patella was found to lateralise increasingly (B-D), which was caused by a concavely shaped patella accompanied by a type B trochlear dysplasia ( $F, G)$

osteotomy of the tibial tubercle was performed, along with a lateral retinacular release on the left knee. Originally, the patient had reported to another department with recurring patellar dislocations in deep knee flexion, which had limited the functionality of her knee since childhood. Contrary to classic patellofemoral instability, no dislocation had ever occurred in the flexion angles of 0 to $30^{\circ}$. Post-operatively, patellar instability persisted and emerging symptoms of anterior knee pain led to further restrictions of the patient's activity level, eventually confining the patient to the use of forearm crutches.

A physical examination revealed a limited active range of motion to $60^{\circ}$ of the knee flexion, as a result of the patient's concerns of an impending dislocation beyond this angle. In passive flexion, a continuous lateral maltracking of the patella was observed from an angle of $30^{\circ}$, leading to further lateralisation with ongoing flexion (Video 1). The right knee presented similar findings of a distal patellar maltracking but with preserved mobility and a full range of motion.

A lateral radiograph indicated a patella infera (CatonDeschamps Index, CDI = 0.74) (Fig. 1A), while standing long-leg radiographs showed a physiological leg axis in the frontal plane (Fig. 1E). Tangential radiographs of the patella in 30,60 and $90^{\circ}$ of knee flexion confirmed the clinical findings of a distal lateral maltracking during the course of progressive knee flexion (Figs. 1B-D). The preoperative computer tomography (CT) showed a trochlear dysplasia (Dejour B) and a conformingly shaped concave patella (Figs. 1F-G). The tibial-tuberosity to trochlear groove (TT-TG) distance was measured to be $16 \mathrm{~mm}$. In a magnetic resonance imaging (MRI) of hips, knees and ankles, normal torsional angles of $-25^{\circ}$ (femoral) and $30^{\circ}$ (tibial) were measured in accordance with Waidelich et al. ${ }^{18}$ The $3 \mathrm{D}$ reconstruction of the $\mathrm{CT}$ showed a lateralised patella that articulated with the lateral condyle, while the trochlea remained empty (Fig. 2).

In the synopsis of all clinical and radiological findings, the patient presented a combination of predisposing anatomical factors, while the distally pronounced maltracking indicated the patella dysplasia to be the main cause for patellofemoral instability. The severe lateral maltracking in this patient was caused by the mismatch between the highly dysplastic patella and the trochlear groove.

\section{Surgical Plan}

Complex cases of patellofemoral instability require a precise preoperative analysis of underlying anatomical risk factors, as well as a careful differentiation between instability and maltracking. ${ }^{5}$ Accordingly, both of these symptoms need to be addressed separately. ${ }^{4}$ In general, patella instability is successfully treated with a reconstruction of the medial patellofemoral ligament (MPFL). ${ }^{19}$

Complementary to the clinical examination, maltracking is reconfirmed in a diagnostic arthroscopy with low or no water pressure at the beginning of the surgery.

To address lateral contractures caused by a preceding lateral release, the lateral arthrotomy can be combined with a lengthening of the lateral retinaculum. ${ }^{20}$

Since, biomechanically, the bony morphology of the patellofemoral joint (PFJ) is a determining factor for patella tracking, 

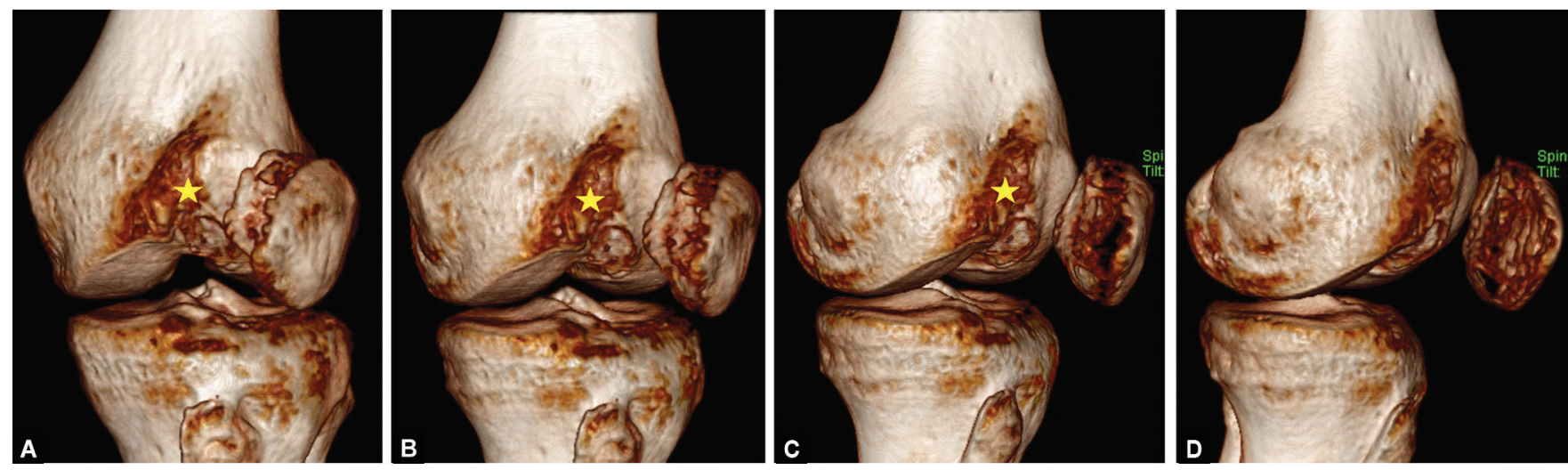

Figs. 2 A to D: A three-dimensional CT reconstruction of the left knee impressively revealed an articulation between the lateral epicondyle and the patella, while the trochlear groove (yellow star) did not participate in the articulation
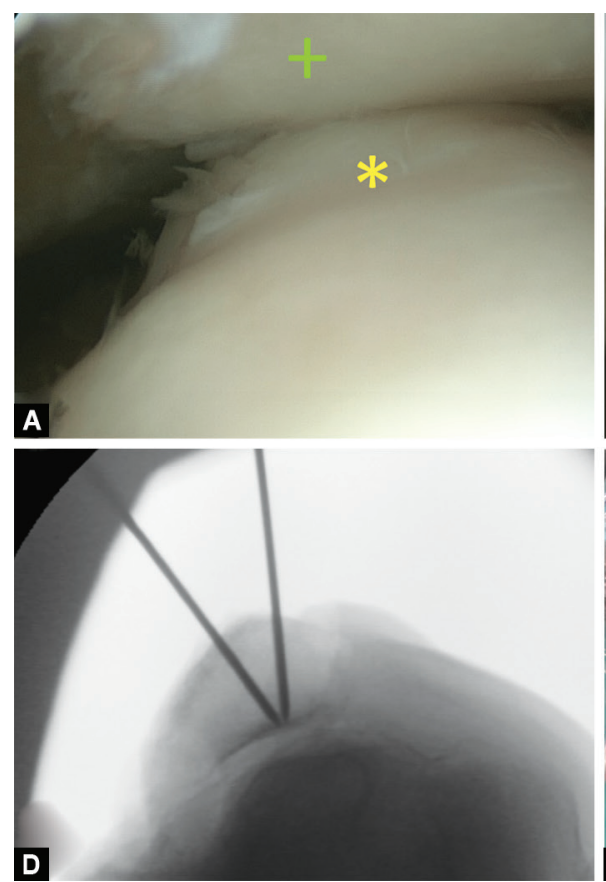

Figs. 3 A to F:The diagnostic arthroscopy revealed a lateralised patella $(+)$, riding on top of the lateral condyle $(*)$, while the trochlea groove remains empty $(A, B)$. Due to the concave shape of the dysplastic patella, its reduction into the trochlear groove was impossible at this point of surgery. The pre-operatively measured and planned wedge was marked with two K-wires and controlled with the image intensifier (C, D). After the bony wedge was removed (green arrow) (E), the osteotomy was closed and osteosynthesis was performed with cannulated screws (F). Subsequently, the patella could be centralised into the trochlear groove. In addition, a medialisation and proximalisation of the tibial tubercle was performed

its correction should be prioritised in this case. ${ }^{7}$ In contrast to a proximal patella maltracking, which is typically observed in trochlear dysplasia, the patella of this patient lateralises distally and in deep flexion. ${ }^{5}$ This section of movement is not addressable by trochleoplasty and the dysplastic shape of the patella presents a high risk of producing incongruent articulation between patella and trochlea. Therefore, after arthroscopic confirmation, an anterior closed-wedge patelloplasty is indicated to reshape the dysplastic patella and to realign patella tracking.

Furthermore, against the background of a borderline increased TT-TG distance of $16 \mathrm{~mm}$, intraoperative reevaluation of patella tracking is indicated, following the patelloplasty. ${ }^{9,21}$ In the case of persisting lateral maltracking, an additional medialisation of the tibial tubercle should be considered, combined with a slight proximalisation in the case of a patella infera. ${ }^{5}$

\section{Surgical Technique}

With the patient in a supine position, the left leg was stored in an electric leg holder and wrapped in a tourniquet. Diagnostic arthroscopy was performed at the beginning of the procedure to reconfirm the indication and to further assess patellofemoral 
tracking (Fig. 3A and Video 2). Even with the manual reduction, it was not possible to re-centre the dysplastic patella into the trochlear groove (Video 3).

The arthrotomy was performed, excising the pre-existing scar, and the patellofemoral joint was exposed. In the process, the superficial and deep layers of the lateral retinaculum were incised in a z-shaped fashion to allow subsequent lengthening of the lateral retinaculum (Fig. 3C).

Under the use of the image intensifier, the wedge was marked with two K-wires in a preoperatively planned angle of $35^{\circ}$ (Fig. $3 \mathrm{C}$ and D). The wedge-osteotomy was performed along the K-wires using an oscillating saw, and the sawn-out wedge was removed (Fig. 3E).

By careful reduction, a physiological convexity of the retropatellar joint surface could be achieved. The osteotomy was secured with a bicortical $3.5 \mathrm{~mm}$ lag screw to close the osteotomy gap and create interfragmentary compression, which was completed by two cannulated $4.0 \mathrm{~mm}$ screws that were drilled in a medial to lateral direction (Fig. 3F). While patella tracking was still lateralised (Fig. 4A), a gentle manual realignment of the patella into the trochlear groove was now possible (Fig. 4B).

In order to further optimise patellar tracking, the tibial tubercle was osteotomised and a $5 \mathrm{~cm}$ bone block was proximalised by $15 \mathrm{~mm}$ and slightly medialised until a physiologically centred patellar tracking was observed (Fig. 4B). The tubercle was fixed with three $3.5 \mathrm{~mm}$ bicortical screws that were drilled in a posteromedial direction to avoid injury of the popliteal vessels (Fig. 4C and D).
Finally, an 18 to $20 \mathrm{~mm}$ gracilis tendon graft was harvested from the ipsilateral pes anserinus and prepared for MPFL reconstruction. A small groove was prepared with a Luer and two suture anchors (FastAk, Arthrex, Naples, FL) that were inserted between the middle and proximal third of the medial patella (Fig. 5A and $B$ ). Under strictly medio-lateral orientation of the image intensifier, the femoral point of isometry was located, a guidewire was inserted and over drilled with a $6 \mathrm{~mm}$ drill. The graft was knotted to the anchors and shuttled underneath the native MPFL through the drill channel using a shuttling suture. The range of motion was tested and the graft was fixed tension-free with a $5 \times 23 \mathrm{~mm}^{2}$ bioabsorbable interference screw (Arthrex, Naples, FL) (Fig. 5C and D).

Finally, during the course of wound closure, the lateral retinaculum was lengthened, adapting the superficial lateral with the deep medial layer. The facia and the subcutaneous tissue were readapted and wound closure was established. Postoperatively, the results were controlled by standard radiographs in two planes (Fig. 5) that were acquired by a CT scan of the right knee (Fig. 6).

\section{Rehabilitation Protocol and Postoperative Results}

The postoperative rehabilitation protocol included a knee brace, allowing full extension and a restricted flexion to $45^{\circ}$ (week 1-3) and $60^{\circ}$ (week 3-6) until the range of motion (ROM) was released. Partial
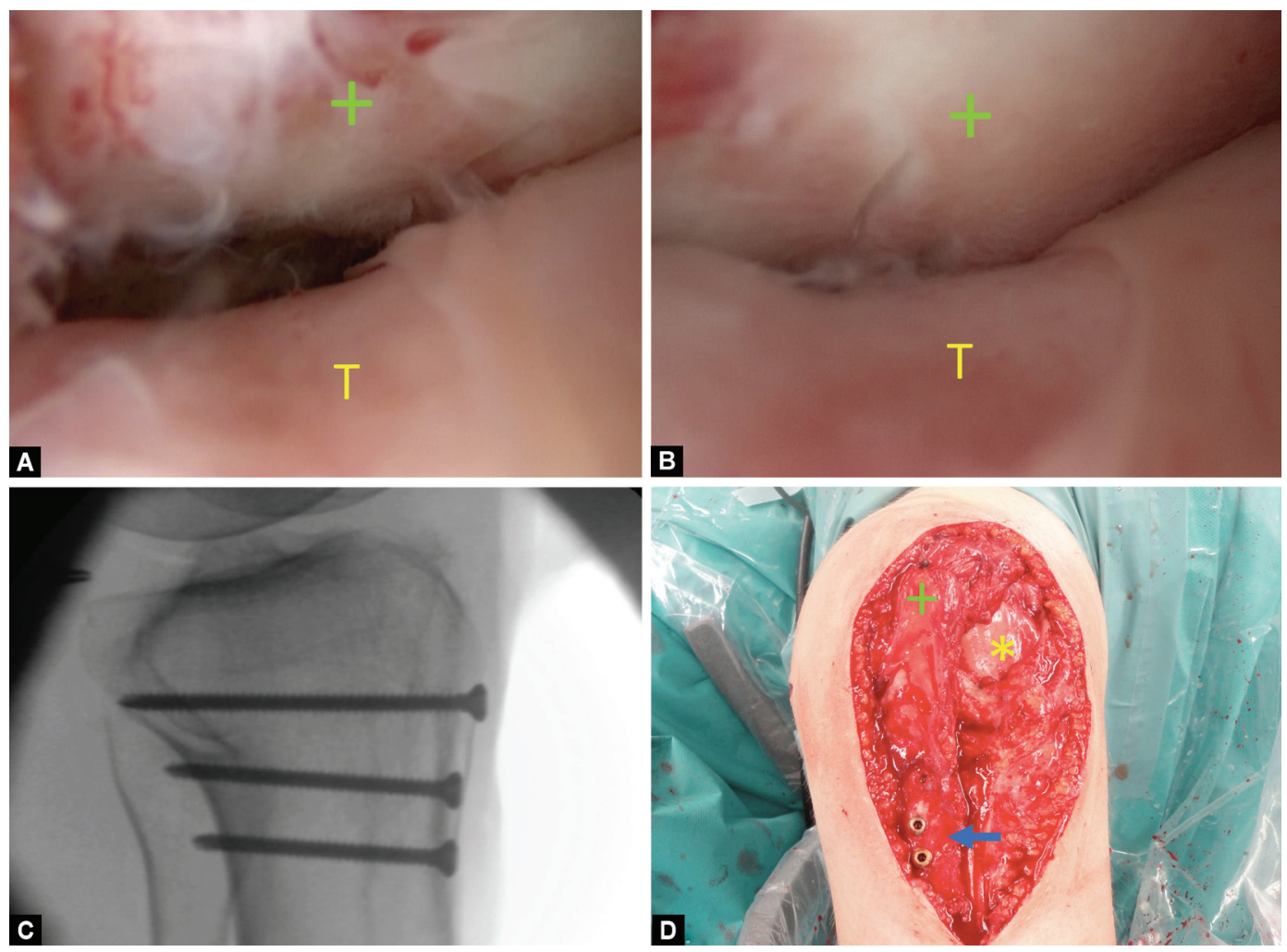

Fig. 4: Arthroscopically, patellar tracking was found to remain slightly lateralized (a), while the patella (+) could easily be reduced into the trochlear groove $(T)$, by applying gentle lateromedial pressure (b). After medializing and proximalizing the tibial tubercle, restoring physiological conditions, it was fixed with three bicortical $3.5 \mathrm{~mm}$ screws (blue arrow) (c, d). The lateral condyle $\left(^{*}\right)$, which previously functioned as the primary counterpart of patellofemoral articulation, was now located lateral to the patellofemoral joint (d) 

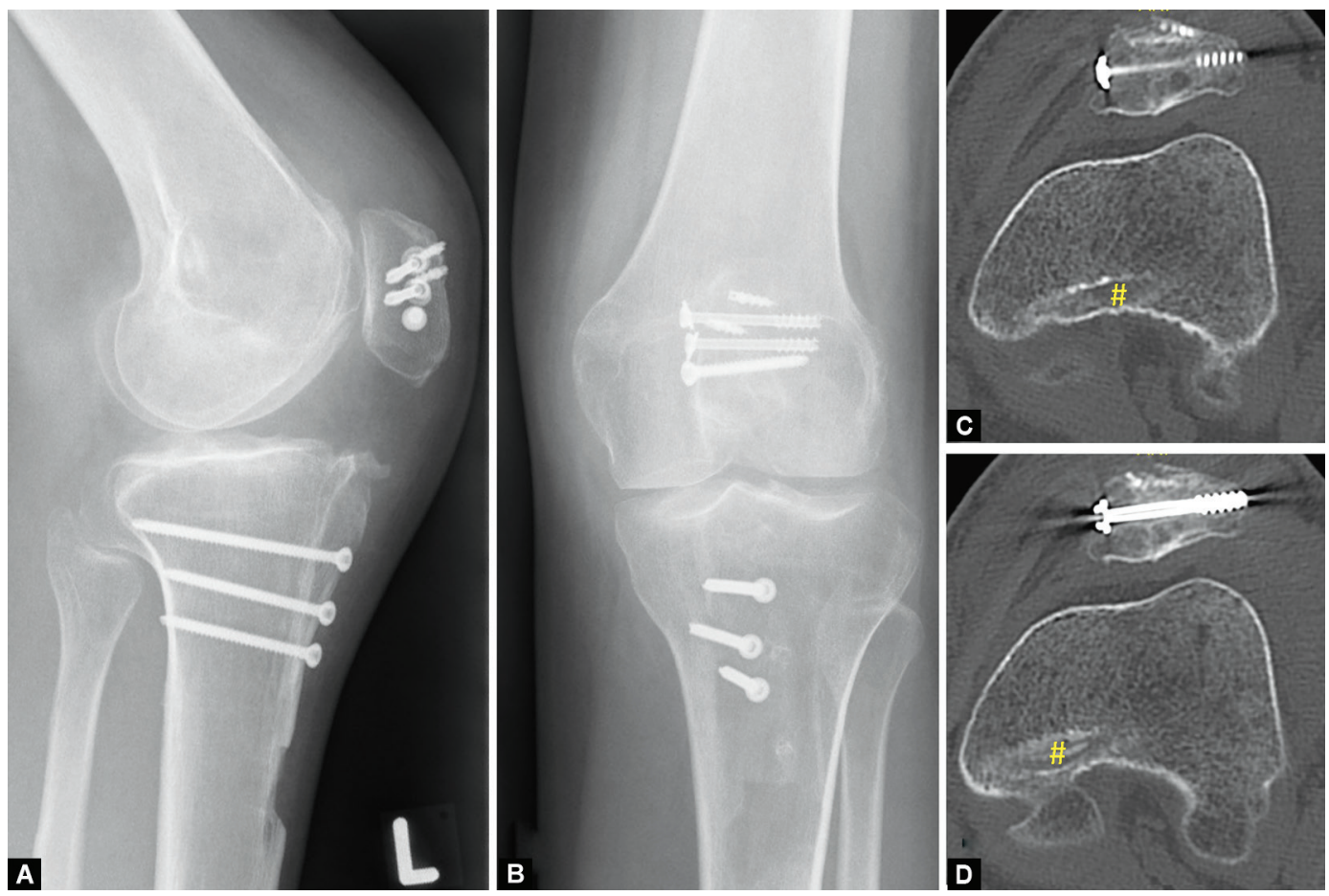

Figs. $5 \mathrm{~A}$ to C: The postoperative radiograph of the left knee in two planes showed an improved patellar height (A) and the correct position of all osteosynthesis materials (B). An additional postoperative CT scan showed the correct position of the femoral drill channel (\#) and demonstrated an improved patellofemoral articulation that was enabled by the newly created conformity of the retropatellar joint surface (C, D)
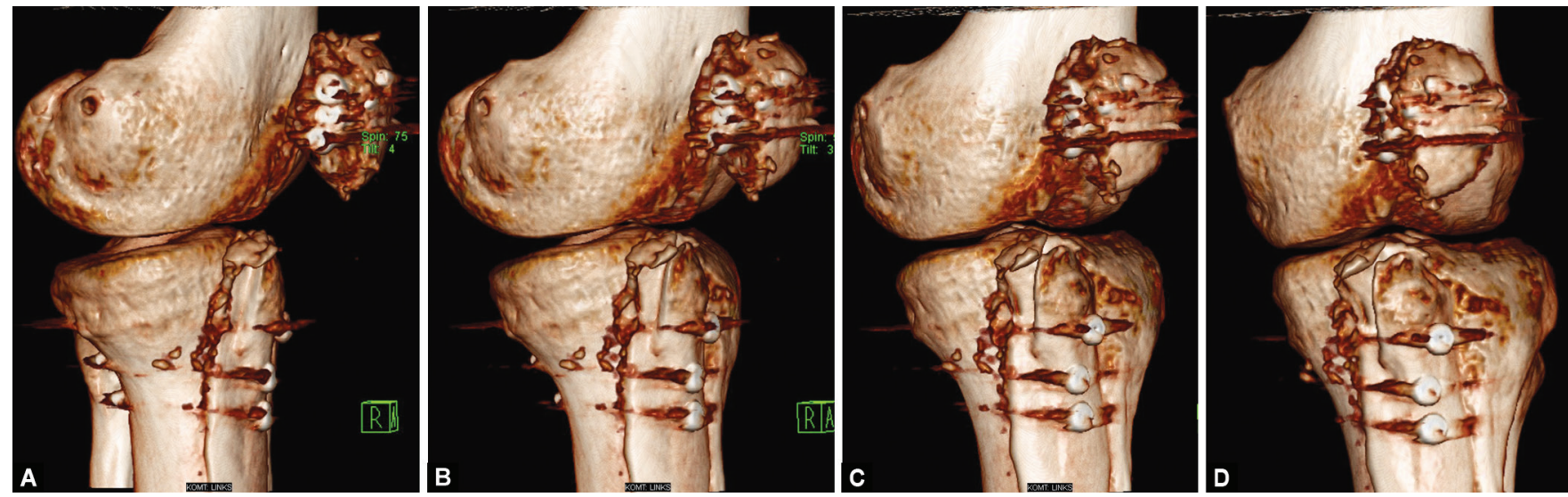

Figs. 6 A to D: A three-dimensional reconstruction of a postoperative CT scan presented a notable improvement of patellofemoral articulation

weight-bearing was prescribed for six weeks. At the 12-month follow-up visit, the patient presented mostly pain-free, and no further dislocations had occurred, allowing her to walk normally without crutches. The active ROM was $0-0-130^{\circ}$, without signs of maltracking (Fig. 7). In the radiological control, the osteotomy site had healed completely.

After 18 months, the patient presented pain free and fully remobilised for the implant removal of the screws. While the preoperative clinical examination revealed a passive ROM of $0-0-145^{\circ}$ (Fig. 8, Video 4), arthroscopic re-evaluation confirmed the findings of a physiological patellar tracking (Fig. 9, Video 5). Knee function presented with a Lysholm score of 90 , as the
Tegner Activity Index improved from 1 (preoperatively) to 3 (postoperatively).

\section{Discussion}

In order to understand patellofemoral maltracking, a thorough preoperative analysis is mandatory, to identify and weigh up all the present anatomical pathologies that can influence patellofemoral kinematics. Through clinical examination, the surgeon also has to differentiate between distal and proximal maltracking. While proximal maltracking may become clinically apparent in terms of the 'reversed J' sign, which describes a sudden re-centring of 

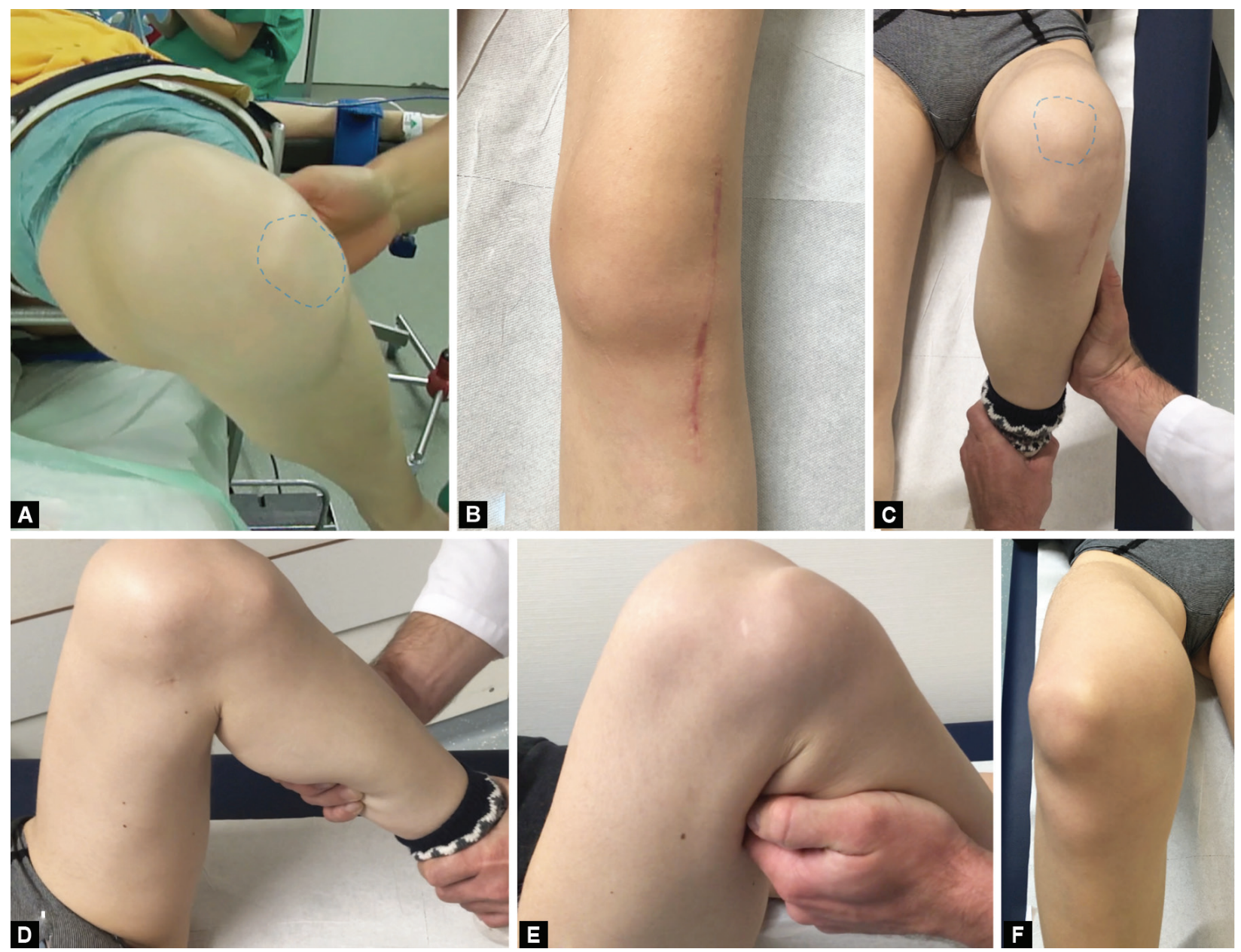

Figs. $7 \mathrm{~A}$ to F: Before the surgery was started, the left knee was re-examined regarding its full range of motion, showing an obvious lateralization of the patella with progressive knee flexion (A). At the 12-month follow-up examination, the wound had healed completely (B) and physiological patellar tracking was restored up to a passive flexion angle of $130^{\circ}(C, D)$. Examination of the contralateral right knee demonstrated similar findings of a distal patellar maltracking $(E, F)$
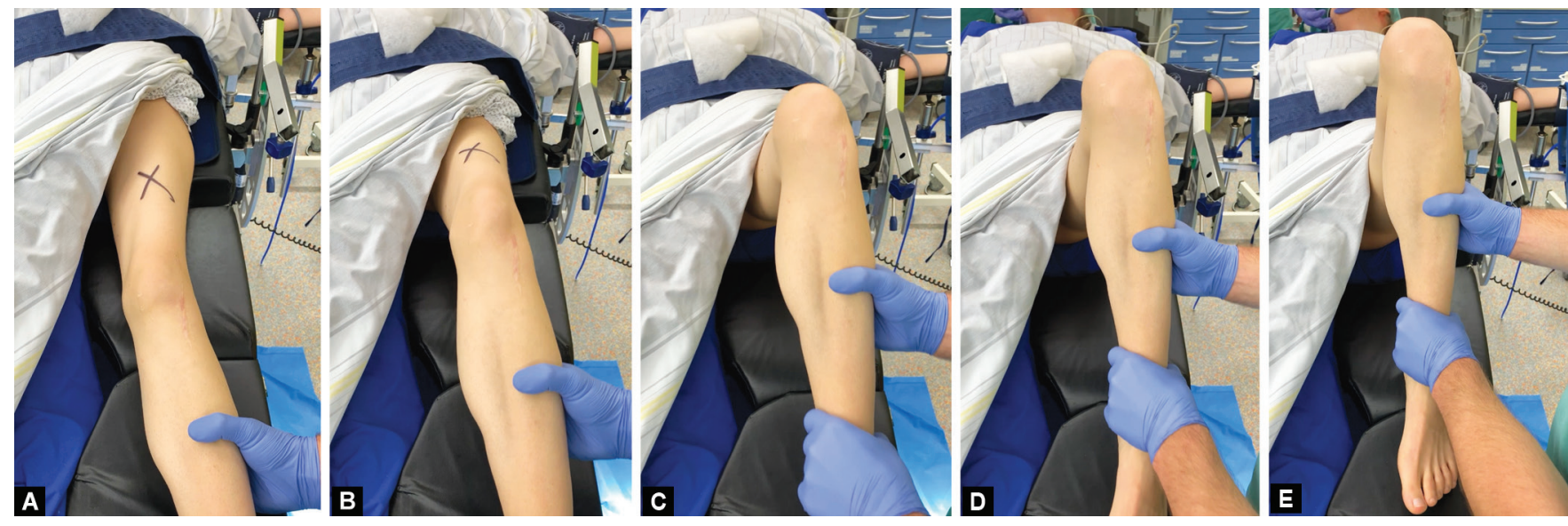

Figs. $8 \mathrm{~A}$ to E: After 18 months, the patient presented for implant removal of the screws. Preoperative patellar tracking during passive range of motion was physiological throughout a range of motion between full extension and $145^{\circ}$ of knee flexion (from left to right) 

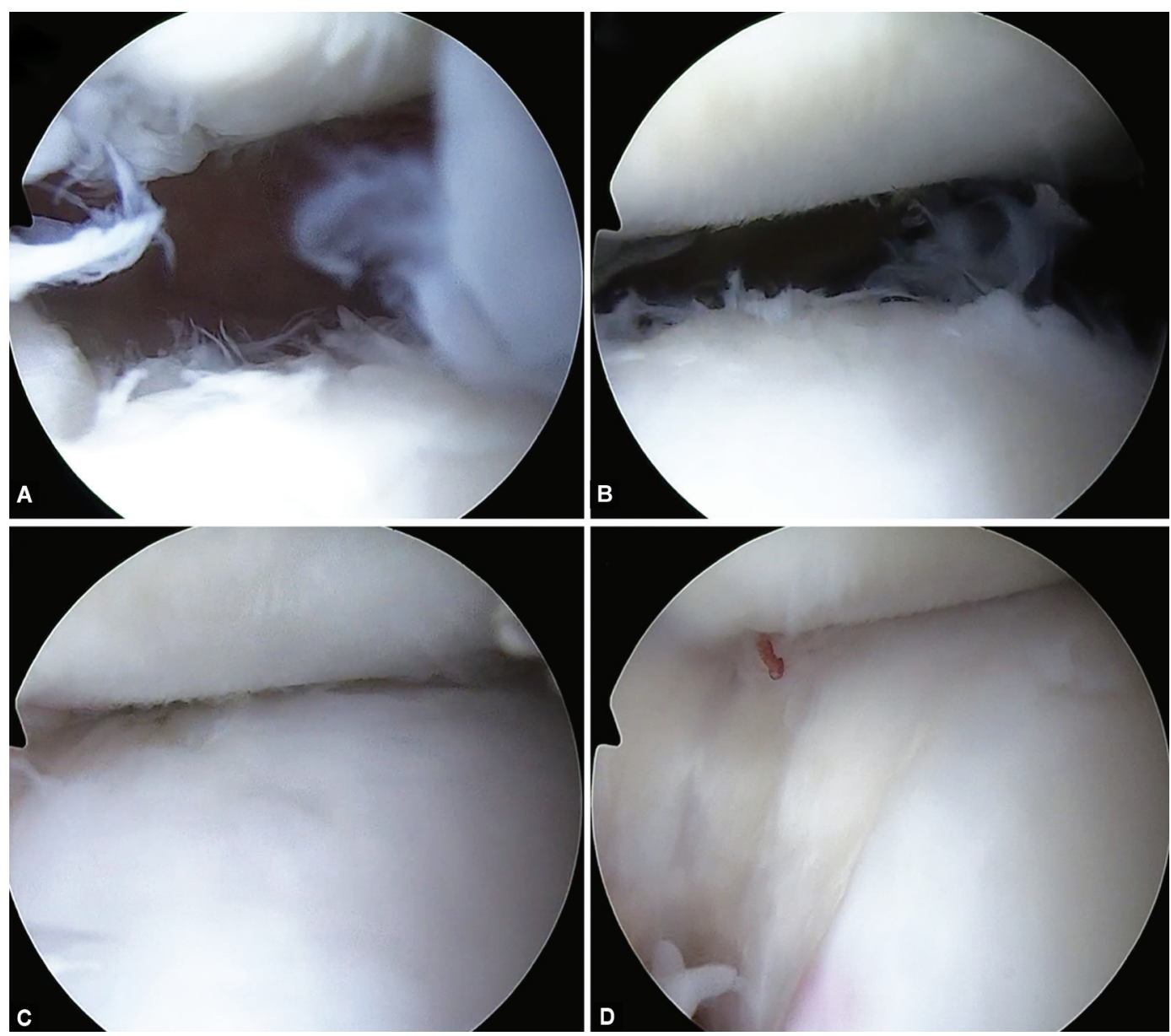

Figs. 9 A to D: The arthroscopic re-evaluation of the patellar tracking at the beginning of the implant removal surgery documented a centralised position of the patella in full extension $(A, B)$, as well as a physiological engagement of the patella into the trochlear groove $(C, D)$

the patella at 20 to $30^{\circ}$ of flexion, distal maltracking occurs when the patella leaves the bony guidance of the trochlear groove at $50-70^{\circ}$ and then slips laterally. ${ }^{5}$ In the presented case, the patella was conformingly shaped to fit its articular counterpart, namely, the physiologically convex-shaped lateral condyle. This led to a constant lateral articulation, which became aggravated as the patella moved distally with increasing flexion, losing the guidance previously provided by the lateral epicondyle.

Although this patient also presented a type B dysplastic trochlea, ${ }^{12}$ a trochleoplasty was not expected to have a beneficial effect as the patella did not articulate with the trochlea at any point of knee flexion. Given the high popularity of this technique, it should also be noted that in cases of a convex trochlear dysplasia, a conformingly shaped patella may present a hidden pitfall when performing a trochleoplasty, thus leading to a sledge-like patellofemoral mismatch.

In the presented case, after the osteotomy of the patella was performed, manual reduction of the patella was achievable again. However, a slight lateral maltracking in motion persisted, most likely caused by a marginally lateralised tibial tuberosity. Although an increased TT-TG of $\geq 16 \mathrm{~mm}$ is not necessarily considered pathological, a tibial tubercle osteotomy (TTO) should be indicated if a simultaneous lateral maltracking is observed..$^{9-11}$
While a growing number of studies have illuminated the influence of anatomical risk factors on patella tracking, most of them are of femoral or tibial origins. ${ }^{3-5,9,14,22}$ Few studies have described patella-altering techniques - mainly lateral facetectomy - for the treatment of isolated lateral patellofemoral osteoarthritis, that is caused by patellar maltracking in native knees. ${ }^{23-27}$ However, to our knowledge, no study has yet outlined the effect of a dysplastic patella on patellofemoral tracking or proposed a suitable treatment option.

Certainly, the described procedure cannot be considered a standard treatment for patients with patella maltracking. The patients need to be selected carefully and special attention should be paid to the exact presentation of patellofemoral tracking. In particular, in cases of a distal lateral displacement of the patella in flexion, the success of proximal realignment procedures can be doubted. Accordingly, a thorough clinical examination is required, supplemented by repetitive pre- and intraoperative re-evaluation, in order to isolate the causative pathology. ${ }^{5}$ In this context, the influence of a dysplastic patella should be considered carefully.

In this case, distal patellofemoral maltracking was caused by a concave patellar dysplasia, which prevented physiological articulation with the trochlear groove. Furthermore, intraoperative observations illustrated that proximal realignment procedures 
alone (TTO) were unable to correct this distal patellar maltracking. So eventually, physiological patellar tracking was re-established by the described technique of patelloplasty.

Although the severity of this rare case is particularly striking, it can be assumed that the influence of the patella is generally underestimated.

\section{LIMITATIONS}

There are some limitations to this report. First of all, the severity in this presented case is rare and the number of patients with comparable pathologies is small. Therefore, the described technique cannot be generalised or recommended for a certain group of patients. Furthermore, the functional outcome and pain reduction depends on the integrity of the patellofemoral cartilage. In our experience, progressive cartilage degeneration presents a limitation to this procedure and may not lead to satisfying results. Also, the technique should be indicated cautiously in the presence of diabetes or nicotine abuse, which may impair bone healing. Finally, the described technique is technically demanding and requires a precise understanding of patellofemoral anatomy and kinematics, as well as a vast experience in the treatment of patellofemoral disorders.

\section{Conclusion}

Although seldomly noted and commonly underestimated, severe cases of patella dysplasia can be associated with a distal patella maltracking and instability. In these cases, closedwedge patelloplasty can be an effective technique for surgical realignment of patella tracking. A thorough preoperative analysis of patellofemoral kinematics is imperative for the correct indication. Further studies with larger numbers of cases will be necessary to evaluate the functional outcome.

\section{ACKnOWLedgements}

The preparation of this manuscript and all described procedures were performed following the ethical standards, in accordance with the Declaration of Helsinki.

\section{References}

1. Christensen TC, Sanders TL, Pareek A, et al. Risk Factors and time to recurrent ipsilateral and contralateral patellar dislocations. Am J Sports Med 2017;45(9):2105-2110. DOI: 10.1177/0363546517704178.

2. Dickschas J, Ferner F, Lutter C, et al. Patellofemoral dysbalance and genua valga: outcome after femoral varisation osteotomies. Arch Orthop Trauma Surg 2018;138(1):19-25. DOI: 10.1007/s00402-0172822-8.

3. Frings J, Krause $M$, Akoto $R$, et al. Clinical results after combined distal femoral osteotomy in patients with patellar maltracking and recurrent dislocations. J Knee Surg 2019;32(9):924-933. DOI: 10.1055/s-0038-1672125.

4. Frings J, Krause M, Akoto R, et al. Combined distal femoral osteotomy (DFO) in genu valgum leads to reliable patellar stabilization and an improvement in knee function. Knee Surg Sports Traumatol Arthrosc 2018;26(12):3572-3581. DOI: 10.1007/s00167-0185000-9.

5. Frosch $\mathrm{KH}$, Schmeling A. A new classification system of patellar instability and patellar maltracking. Arch Orthop Trauma Surg 2016;136(4):485-497. DOI: 10.1007/s00402-015-2381-9.

6. Hevesi $\mathrm{M}$, Heidenreich $\mathrm{MJ}, \mathrm{Camp} \mathrm{CL}$, et al. The recurrent instability of the patella score: a statistically based model for prediction of long-term recurrence risk after first-time dislocation. Arthroscopy 2019;35(2):537-543. DOI: 10.1016/j.arthro.2018.09.017.

7. Frings J, Balcarek P, Tscholl PM, et al. Conservative versus surgical treatment for primary patellar dislocation-a systematic review to guide risk stratification. Dtsch Arztebl Int 2020;117(16):279-286. DOI: 10.3238/arztebl.2020.0279.

8. Magnussen RA, De Simone V, Lustig S, et al. Treatment of patella alta in patients with episodic patellar dislocation: a systematic review. Knee Surg Sports Traumatol Arthrosc 2014;22(10):2545-2550. DOI: 10.1007/s00167-013-2445-8.

9. Frings J, Krause M, Wohlmuth $P$, et al. Influence of patient-related factors on clinical outcome of tibial tubercle transfer combined with medial patellofemoral ligament reconstruction. Knee 2018;25(6):1157-1164. DOI: 10.1016/j.knee.2018.07.018.

10. Schoettle PB, Zanetti M, Seifert B, et al. The tibial tuberosity-trochlear groove distance; a comparative study between $\mathrm{CT}$ and MRI scanning. Knee 2006;13(1):26-31. DOI: 10.1016/j.knee.2005.06.003.

11. Franciozi CE, Ambra LF, Albertoni LJB, et al. Anteromedial tibial tubercle osteotomy improves results of medial patellofemoral ligament reconstruction for recurrent patellar instability in patients with tibial tuberosity-trochlear groove distance of 17 to $20 \mathrm{~mm}$. Arthroscopy 2019;35(2):566-574. DOI: 10.1016/j.arthro.2018.10.109.

12. Dejour H, Walch G, Nove-Josserand L, et al. Factors of patellar instability: an anatomic radiographic study. Knee Surg Sports Traumatol Arthrosc 1994;2(1):19-26. DOI: 10.1007/BF01552649.

13. Nelitz M, Dreyhaupt J, Lippacher S. Combined trochleoplasty and medial patellofemoral ligament reconstruction for recurrent patellar dislocations in severe trochlear dysplasia: a minimum 2-year follow-up study. Am J Sports Med 2013;41(5):1005-1012. DOI: 10.1177/0363546513478579.

14. Dickschas J, Harrer J, Pfefferkorn R, et al. Operative treatment of patellofemoral maltracking with torsional osteotomy. Arch Orthop Trauma Surg 2012;132(3):289-298. DOI: 10.1007/s00402-0111303-8.

15. Wilberg G. Roentgenographic and anatomic studies on the femoropatellar joint. Acta Orthop Scand 1941;12(1-4):319-410. DOI: 10.3109/17453674108988818.

16. Figueroa-Silva $O$, Vicente $A$, Agudo $A$, et al. Nail-patella syndrome: report of 11 pediatric cases. J Eur Acad Dermatol Venereol 2016;30(9):1614-1617. DOI: 10.1111/jdv.13683.

17. Morscher E. Osteotomy of the patella in chondromalacia. Preliminary report. Arch Orthop Trauma Surg 1978;92(2-3):139-147. DOI: 10.1007/ BF00397950.

18. Waidelich H, Strecker W, Schneider E. [Computed tomographic torsion-angle and length measurement of the lower extremity. The methods, normal values and radiation load]. RoFo 1992;157(3):245251. DOI: $10.1055 / \mathrm{s}-2008-1033007$.

19. Schottle PB, Fucentese SF, Romero J. Clinical and radiological outcome of medial patellofemoral ligament reconstruction with a semitendinosus autograft for patella instability. Knee Surg Sports Traumatol Arthrosc 2005;13(7):516-521. DOI: 10.1007/s00167-0050659-0.

20. Pagenstert G, Wolf N, Bachmann M, et al. Open lateral patellar retinacular lengthening versus open retinacular release in lateral patellar hypercompression syndrome: a prospective doubleblinded comparative study on complications and outcome. Arthroscopy 2012;28(6):788-797. DOI: 10.1016/j.arthro.2011. 11.004.

21. Balcarek $P$, Oberthur $S$, Hopfensitz $S$, et al. Which patellae are likely to redislocate? Knee Surg Sports Traumatol Arthrosc 2014;22(10):23082314. DOI: 10.1007/s00167-013-2650-5.

22. Elias JJ, Jones KC, Rezvanifar SC, et al. Dynamic tracking influenced by anatomy following medial patellofemoral ligament reconstruction: computational simulation. Knee 2018;25(2):262-270. DOI: 10.1016/j. knee.2018.02.002.

23. Ferrari MB, Sanchez G, Chahla J, et al. Arthroscopic patellar lateral facetectomy. Arthrosc Tech 2017;6(2):e357-e362. DOI: 10.1016/j. eats.2016.10.002. 
24. Jones RE, Rumack WA. Arthroscopic facetectomy for severe isolated patellofemoral arthrosis. Orthopedics 2008;31(9):917-919. DOI: 10.3928/01477447-20080901-18.

25. Montserrat F, Alentorn-Geli E, Leon V, et al. Partial lateral facetectomy plus Insall's procedure for the treatment of isolated patellofemoral osteoarthritis: survival analysis. Knee Surg Sports Traumatol Arthrosc 2014;22(1):88-96. DOI: 10.1007/s00167-012-2286-x.
26. Sanchis-Alfonso V, Koh JL. Joint-preserving osteotomies for isolated patellofemoral osteoarthritis: alternatives to arthroplasty. Am J Orthop (Belle Mead NJ) 2017;46(3):139-145.

27. Wu T, Tang S, Wang F. Treatment for lateral patellar impingement syndrome with arthroscopic lateral patelloplasty: a bidirectional cohort study. J Orthop Surg Res 2017;12(1):173. DOI: 10.1186/s13018017-0676-y.

Video 1: With the patient in general anaesthesia, preoperative examination of the left knee shows increasing lateralisation of the patella during continuous passive flexion

Video 2: A lateralised, concavely shaped patella is riding on top of the lateral condyle, while an empty trochlear groove can be observed. A manual reduction of the patella into the trochlear groove is prevented by the dysplastic shape of the patella, leading to a mismatch between patella and trochlea groove

Video 3: Due to the severe dysplasia of the patella, manual reduction of the patella into the trochlear groove was not possible

Video 4: The dynamic preoperative examination after 18 months documented a physiological patellar tracking without further lateral displacement of the patella during the range of motion

Video 5: Arthroscopically, after 18 months, the repetitive examination of dynamic patellofemoral articulation revealed that the patella remained centred inside the trochlear groove throughout the range of motion 